Subscriber access provided by UNIV NEW ORLEANS

\title{
Communication
}

\section{Bottom-up design of three-dimensional carbon-honeycomb with superb specific strength and high thermal conductivity}

\author{
Zhenqian Pang, Xiaokun Gu, Yujie Wei, Ronggui Yang, and Mildred S. Dresselhaus
}

Nano Lett., Just Accepted Manuscript • DOI: 10.1021/acs.nanolett.6b03711 • Publication Date (Web): 05 Dec 2016

Downloaded from http://pubs.acs.org on December 5, 2016

\section{Just Accepted}

"Just Accepted" manuscripts have been peer-reviewed and accepted for publication. They are posted online prior to technical editing, formatting for publication and author proofing. The American Chemical Society provides "Just Accepted" as a free service to the research community to expedite the dissemination of scientific material as soon as possible after acceptance. "Just Accepted" manuscripts appear in full in PDF format accompanied by an HTML abstract. "Just Accepted" manuscripts have been fully peer reviewed, but should not be considered the official version of record. They are accessible to all readers and citable by the Digital Object Identifier (DOI®). "Just Accepted" is an optional service offered to authors. Therefore, the "Just Accepted" Web site may not include all articles that will be published in the journal. After a manuscript is technically edited and formatted, it will be removed from the "Just Accepted" Web site and published as an ASAP article. Note that technical editing may introduce minor changes to the manuscript text and/or graphics which could affect content, and all legal disclaimers and ethical guidelines that apply to the journal pertain. ACS cannot be held responsible for errors or consequences arising from the use of information contained in these "Just Accepted" manuscripts. 


\title{
Bottom-up design of three-dimensional carbon-honeycomb with superb specific strength and high thermal conductivity
}

\author{
Zhenqian Pang ${ }^{1 \dagger}$, Xiaokun $\mathrm{Gu}^{2 \dagger}$, Yujie Wei ${ }^{1 \star}$, Ronggui Yang $^{{ }^{*}}$, Mildred S. Dresselhaus ${ }^{3^{*}}$ \\ ${ }^{1}$ LNM, Institute of Mechanics, Chinese Academy of Sciences, Beijing 100190, China \\ ${ }^{2}$ Department of Mechanical Engineering and Materials Science and Engineering \\ Program, University of Colorado, Boulder, CO 80309, USA \\ ${ }^{3}$ Department of Physics and EECS, Massachusetts Institute of Technology, Cambridge, \\ MA 02139, USA \\ ${ }^{\dagger}$ Co-first author, these authors contributed equally to this work. \\ ${ }^{*}$ Correspondence: yujie_wei@Inm.imech.ac.cn \\ ronggui.yang@colorado.edu \\ millie@mgm.mit.edu
}

\begin{abstract}
:
Low-dimensional carbon allotropes, from fullerenes, carbon nanotubes, to graphene, have been broadly explored due to their outstanding and special properties. However, there exist significant challenges in retaining such properties of basic building blocks when scaling them up to three-dimensional materials and structures for many technological applications. Here we show theoretically the atomistic structure of a stable 3-dimensional carbon honeycomb (C-honeycomb) structure with superb mechanical and thermal properties. A combination of $\mathrm{sp}^{2}$ bonding in the wall and $\mathrm{sp}^{3}$ bonding in the triple junction of C-honeycomb is the key to retain the stability of C-honeycomb. The specific strength could be the best in structural carbon materials, and this strength remains at a


high level but tunable with different cell sizes. C-honeycomb is also found to have a very high thermal conductivity, e.g. $>100 \mathrm{~W} / \mathrm{mK}$ along the axis of the hexagonal cell with a density only $\sim 0.4 \mathrm{~g} / \mathrm{cm}^{3}$. Due to the low density and high thermal conductivity, the specific thermal conductivity of C-honeycombs is larger than most engineering materials, including metals and high thermal conductivity semiconductors, as well as light-weight CNT arrays and graphene-based nanocomposites. Such high specific strength, high thermal conductivity, and anomalous Poisson's effect in C-honeycomb render it appealing for the use in various engineering practices.

\section{Keywords:}

Carbon honeycomb, specific strength, thermal conductivity, $\mathrm{sp}^{3}$-bonding 
Inspired by the extraordinary properties seen in low-dimensional carbon allotropes, such as carbon nanotubes and graphene, researchers are exploring ways to realize stable carbon structures of different kinds ${ }^{1}$. At the same time, it is desirable to use such low-dimensional carbon structures as building blocks to realize three-dimensional (3-D) engineering materials and structures which may inherit their superb properties. In reality, the scale-up leads to a substantial degradation of properties that we desire to retain. Mechanically, single-layer graphene ${ }^{2}$ is the strongest materials with an in-plane modulus of about $1 \mathrm{TPa}$ and a tensile strength of $130 \mathrm{GPa}^{3}$. The in-plane scale-up by chemically growing large-area polycrystalline graphene ${ }^{4}$ is indeed very successful: the strength of polycrystalline graphene may be as high as that of pristine graphene ${ }^{5}$. Even though different boundary structures and pre-existing defects may reduce the strength dramatically $^{6-8}$, two-dimensional polycrystalline graphene may have a strength on the order of tens of GPa, a value significantly greater than for most existing engineering materials. However, the realized mechanical and thermal properties of 3-D carbon materials, by staggering graphene sheets or vertically grown carbon nanotube arrays, are significantly lower than those of individual graphene sheets or individual CNTs ${ }^{9}$ : the strongest graphene paper reported in the literature has a strength $2 \sim 3$ orders of magnitude lower than that of graphene ${ }^{10}$. Similarly, the thermal conductivity of a single carbon nanotube was reported to be more than $3000 \mathrm{~W} / \mathrm{mK}^{11}$, while the best efforts on vertically grown carbon nanotube arrays for thermal interface materials resulted in a thermal conductivity of two orders of magnitude lower ${ }^{12}$. The huge gap in the thermal and mechanical properties between the low-dimensional carbon allotropes and their 3-D derivatives originates from the dissimilar bonding characteristics between carbon atoms 
within graphene or CNTs and the architected 3-D engineering materials: The intra-structure bonding is covalent in nature, while van der Waals bonding dominates between different layers/tubes or with other materials ${ }^{1}$. Such heterogeneous bonding leads to property inheritance a mission impossible.

The interest in finding 3-D carbon structures has been lasting for decades. ${ }^{13-21}$ While the stability and phase transition of bulk carbon allotropes have widely explored, ${ }^{22-24}$ the stability of the hypothesized 3-D carbon structures remains an open question. Furthermore, the elastic constants of some of these hypothesized 3-D carbon structures were studied, but due to the connections among different graphene pieces, whether the exceptional properties, such as large strength and high thermal conductivity can be inherited by these structures is unclear. Recent success in the synthesis of carbon honeycomb (C-honeycomb) ${ }^{25}$ shows a great potential in scaling up the low-dimensional carbon allotropes to 3-D engineering materials and structures while retaining strong covalent bonding. Such a C-honeycomb structure may circumvent the change of bonding while using graphene as basic building blocks. The junctions that connect graphene layers to form honeycomb walls, as shown in Figure 1a, are hence crucial for the physical properties of C-honeycombs.

These materials can find applications as conducting framework for electrodes in lithium ion batteries and fuel cells, supercapacitors, membranes for water treatment and reclamation, and so on. Krainyukova and Zubarev ${ }^{25}$ postulated that the C-honeycomb structure is made by connecting graphene nanoribbons along their zigzag edges and the carbon atoms in the junction line are uniformly distributed and each such carbon atom is connected with three neighbouring carbon atoms that belong to three graphene ribbons, 
respectively, through $\mathrm{sp}^{2}$ bonding, as shown in the Supporting Information (Figure S1). However, there is no theoretical or experimental evidence to verify such an assumption. Furthermore, whether the C-honeycomb could successfully inherit the outstanding properties of two-dimensional single-layer graphene remains an open question. In this report, we employ first-principles density-functional theory (DFT) calculations to study the structural stability of the postulated C-honeycombs. We reveal a stable C-honeycomb structure where the junctions are indeed formed by $\mathrm{sp}^{3}$ bonding. Such a stable C-honeycomb structure exhibits the highest specific strength and thermal conductivity among all architected 3-D carbon materials that might find applications in ultra-light structural and multifunctional materials.

First-principles DFT calculations are performed to determine the atomic structure of C-honeycomb using the Vienna Ab initio Simulation Package (VASP). ${ }^{26,27}$ The projector augmented wave (PAW) pseudopotentials ${ }^{28}$ and the generalized gradient approximation (GGA) of the Perdew-Burke-Ernzerhof (PBE) functional ${ }^{29}$ are used. The C-honeycomb structures studied here are relaxed using a conjugate gradient (CG) algorithm until the atomic forces are converged to $1 \mathrm{e}^{-5} \mathrm{eV} / \AA$ and the total energy is minimized. To examine the stability, phonon dispersion of C-honeycomb structures is computed using Phonopy package ${ }^{30}$ and the ab initio molecular dynamics simulation are conducted. The parameters used in these simulations are documented in the Supporting Information section I (SI-1). 

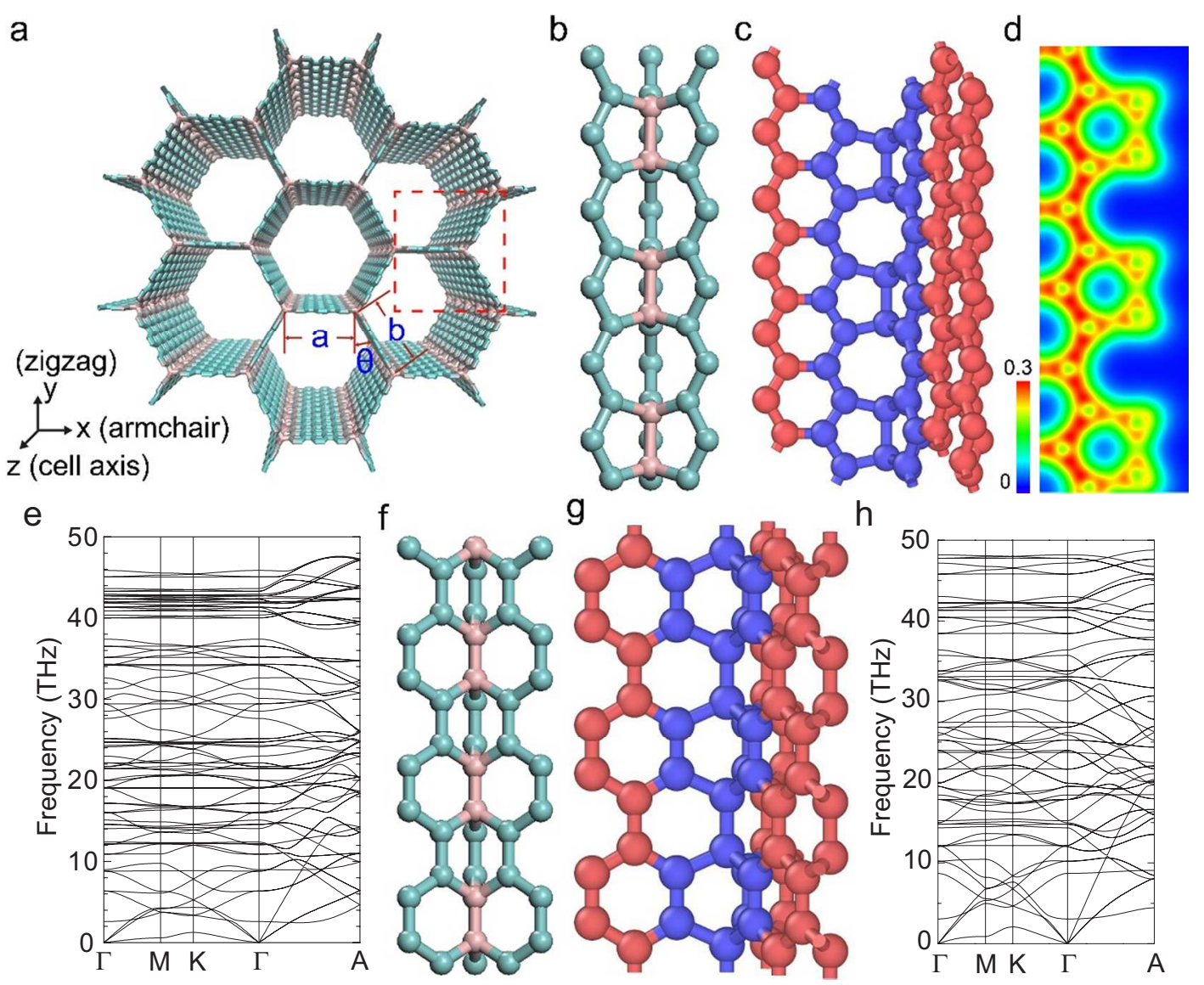

Figure 1. Stable C-honeycomb structure. (a) Atomistic structure of carbon honeycomb and the coordinate defined based on the honeycomb; (b) and (c) Local atomistic structure at the 5-5-8 junction of C-honeycomb cells viewed along the armchair direction and viewed along the zigzag direction, respectively, and (d) the electron density at the junction region (only one-third portion of junction is shown due to the symmetry); (e) The phonon dispersion of the stable C-honeycomb with cell size of $5.8 \AA$ where all phonons have positive frequencies. (f) to $(\mathrm{h})$ The 6-6-6 junction along armchair direction of graphene wall: (f) and (g) Local atomistic structure at the 6-6-6 junction of C-honeycomb; (h) The phonon dispersion of the stable C-honeycomb with cell size of $5.2 \AA$ where all phonons have positive frequencies.

Depending on whether the widths of graphene nanoribbons are identical or not, the C-honeycomb structure can be ordered with the same size of a and b, as shown in Figure $1 \mathrm{a}$, or irregular with different size of $\mathrm{a}$ and $\mathrm{b}$. For simplicity we focus on only the ordered C-honeycombs, where the cell sizes, a and b, are the same under an unstrained condition. Figure $1 \mathrm{~b}$ and $\mathrm{c}$ show the zoom-in views of a fraction of a C-honeycomb, 
including the junction region and three graphene nanoribbons. In what follows, we term a junction based on its minimum periodic defective structure. The junction in Fig. $1 \mathrm{~b}$ is hence termed as 5-5-8 junction since it is composed of an array of defective units, and each containing two 5-rings and one 8-ring. As shown, the cell size, a (and b), can be tuned by changing the width of the graphene nanoribbons, with results in a C-honeycomb with a different density. We start with an unstrained C-honeycomb, whose cell sizes, $a$ and $b$, are set to be $5.8 \AA$, with the postulated zigzag junction structure. ${ }^{25}$ Imaginary phonon frequencies shown in Figure S1d suggest that the C-honeycombs with such proposed junctions ${ }^{25}$ are mechanically unstable. The instability of structure shown in Figure S1a (termed 5-5 junction) can be understood by the nature of $\mathrm{sp}^{2}$ bonding, which tends to form a smooth structure, as those found in both graphene and carbon nanotubes. Here the graphene nanoribbon plane is perpendicular to the fragment consisting of the atom in the junction line and its neighboring atoms. Such a distortion from a planar structure destroys the parallel orientation of the constituent $p$ orbitals. For the atoms in the junctions, they would prefer to form $\mathrm{sp}^{3}$ bonding with their neighbors for lower energy level. Such hybridized $\mathrm{sp}^{2} / \mathrm{sp}^{3}$ bonding may give rise to other stable two-dimensional structures as well. ${ }^{31}$

To understand the stability of C-honeycomb structures, we also ran ab initio molecular dynamics simulations on the C-honeycombs with 5-5-8 junction and 5-5 junction under constant temperature and volume ensemble at $300 \mathrm{~K}$ to observe the evolution of the carbon atoms. As expected, the 5-5-8 junction is stable but the 5-5 junction structure cannot be maintained. Carbon atoms in the 5-5 junction deviate from their initial positions by moving closer to form pairs, which is the fundamental difference 
between the unstable structure and the structure revealed from the simulation. The junction we observed here is essentially identical to the proposed "graphitic $\mathrm{Y}$ junctions" by Kawai et al. ${ }^{15}$

As shown in Figure $1 \mathrm{~b}$ and $\mathrm{c}$, in the current structure, each carbon atom in the junction connects with four neighboring atoms, one in the junction line and the other three in the three neighboring graphene nanoribbons, indicating that carbon atoms have formed $\mathrm{sp}^{3}$ bonds in the 5-5-8 junction. The contour of the electron density is plotted in Figure $1 \mathrm{~d}$ to further confirm the forming of bonds between two neighboring carbon atoms in the 5-5-8 junction line. In fact, the formation of $\mathrm{sp}^{3}$ bonding is quite similar to the phase transition from graphite to diamond when the interlayer graphite is compressed to such a short interatomic distance.

The cohesive energy of the stable C-honeycomb is $-7.768 \mathrm{eV} /$ atom, 0.135 $\mathrm{eV} /$ atom lower than that with purely $\mathrm{sp}^{2}$ bonding. While the cohesive energy of such a C-honeycomb structure is higher than that in graphene, we also note that the stability of the structure is not solely governed by the cohesive energy, as evident from the significant distinction in cohesive energy among carbon allotropes. The key to the stability of a structure is indeed that there exists sufficiently high energy barrier (to the unstable state) from a local energy minimum to resist thermal perturbation. From the calculated phonon dispersion of the C-honeycomb structures with $\mathrm{sp}^{3}$ bonding at the 5-5-8 junction, as shown in Figure 1e, one could expect that the current C-honeycomb is stable since all phonon frequencies are positive.

The evolution from the unstable structure to the stable structure could be explained by the following mechanism. The single atoms in the 5-5 junction lines form 
$\mathrm{sp}^{2}$ bonds with their neighboring atoms, and their unhybridized $\mathrm{p}$-orbitals are directed along the junction line. The interaction between these orbitals is similar to those between different sheets in graphite. Due to the short interatomic distance $(\sim 2.5 \AA)$ compared with the interlayer distance in graphite, those atoms are expected to repel with each other, and would prefer to form pairs, instead of staying in their original positions as single atoms in an unstable equilibrium state.

The 5-5-8 junction has alternative C-C bonds shared by three 5 -rings of the walls. There is a vacancy formed by the three 8-rings along the cell-axis of the junction (Figure $1 b$ and $c)$.

We also construct C-honeycomb with graphene nanoribbons connected along their armchair edges. Such a junction is composed of periodic units containing two coplanar 6-atom rings and one non-coplanar 6-atom ring (Figure $1 \mathrm{f}$ and $\mathrm{g}$ ), and is termed as 6-6-6 junction. The structure was hypothesized by Park and $\mathrm{Ihm},{ }^{21}$ and Kuc and Seifert. ${ }^{16}$ We calculated the phonon dispersion of the $C$-honeycomb with $a=b=5.2 \AA$. The result shown in Figure $1 \mathrm{~h}$ indicates that it is mechanically stable as well.

We now explore the mechanical and thermal properties of the stable C-honeycomb. To study the mechanical properties of carbon honeycomb, the quasi-static displacement-controlled deformation is used by imposing the deformation with a small strain increment (a typical value of 0.01 is used) along one direction. The other two dimensions of the simulation domain, as well as the atomic coordinates, are adjusted through CG algorithm to make the total energy minimized. The corresponding stress is then obtained from the reaction force induced by the imposed strain divided by the current cross-sectional area. Molecular dynamics (MD) simulations are performed to 
analyze the thermal conductivity. While recently the first-principles-based Boltzmann transport equation method has been widely used to predict the thermal conductivity of many crystalline materials, and the calculated thermal conductivity is usually consistent with experimental measurements, it can be challenging to apply such methodology to materials with complicated structures due to the computational cost. Thus, we perform equilibrium MD simulations using LAMMPS ${ }^{32}$ to estimate the thermal conductivity of carbon honeycombs through Green-Kubo formalism. ${ }^{33}$ Although in classical MD simulations the quantum effects are not included, the thermal conductivity from molecular dynamics simulations is very close to that from the Boltzmann transport equation calculations where the quantum effects are fully taken into account (within $15 \%),{ }^{34}$ as well as the measured data, even for graphene, whose Debye temperature is around $2000 \mathrm{~K}$. The optimized reactive empirical bond-order potential ${ }^{34}$ is employed to describe the interatomic interactions among carbon atoms. The reported thermal conductivity is obtained from the averaged value from ten independent runs. The details of mechanical calculations and thermal conductivity simulations are presented in the Supporting Information SI-II and SI-III, respectively.

As both the 5-5-8 junction and the 6-6-6 junction C-honeycomb exhibit similar mechanical behavior, we will focus on the 5-5-8 junction in what follows. The corresponding mechanical properties shown in Figure 1b. For convenience, we define a coordinate system based on the C-honeycomb structure, as illustrated in Figure 1a. It is noted that the zigzag direction of graphene layers in each wall of the c-honeycomb is perpendicular to the armchair direction of the C-honeycomb. Unless stated otherwise, for the rest of this work, the armchair and the zigzag direction are referred to the 3-D 
C-honeycomb structure that defined in Figure 1a. The mechanical properties of C-honeycombs are studied by DFT calculations, with detailed information supplied in SI-2. Figure 2 shows the mechanical behavior of the C-honeycomb subjected to tension along different directions. The stress-strain curves of C-honeycomb of different cell sizes are shown in Figure 2a, with the loading along the armchair direction on the left, the loading along the zigzag direction in the middle and the loading along the cell-axis on the right. With the minimum cell size $\mathrm{a}=5.8 \AA$, the $\mathrm{C}$-honeycomb structure shown in Figure 1 has a strength of $37.8 \mathrm{GPa}$ along the armchair direction (x-axis) and $38.6 \mathrm{GPa}$ in the zigzag direction (y-axis). When the cell size increases to $a=22.9 \AA$, the respective strengths are about $0.9 \mathrm{GPa}$ and $8 \mathrm{GPa}$. Interestingly, the stress-strain response of C-honeycombs is very nonlinear, with growing Young's modulus when strained at the initial stage. We show in Figure S4 the evolution of Young's modulus as a function of strain for C-honeycombs of different sizes and loading. At the continuum level, the Young's moduli $E_{z z}$ and $E_{a c}$ of a C-honeycomb in the zigzag and armchair direction are given as $^{35} \frac{E_{z Z}}{B_{m}}=\left(\frac{1}{12 b^{3}}\right) \frac{\cos \theta}{(a / b+\sin \theta) \sin ^{2} \theta}$ and $\frac{E_{a c}}{B_{m}}=\left(\frac{1}{12 b^{3}}\right) \frac{(a / b+\sin \theta)}{\cos ^{3} \theta}$, respectively. Here $B_{m}$ is the normal bending stiffness of the graphene wall ${ }^{36}$, and $a, b, \theta$ are the three geometrical parameters of the C-honeycomb (Figure 1a). The theoretical predictions in both the armchair and zigzag direction of the C-honeycomb before deformation are also shown in Figure S4. This nonlinear elasticity is a result of strongly coupled tension and bending deformation to the graphene nanoribbon wall. The patterns of the deformed C-honeycomb cell size $a=b=10.1 \AA$ at different strains suggest that the angle between the honeycomb walls changes dramatically as loading increases (Figure S5). Atoms within a wall are not coplanar in most cases. Such pre-curved graphene walls may 
introduce nonlinear mechanical response during tension. With growing cell size, both the strength and the failure strain decrease, as seen in Figure $2 \mathrm{~b}$. While the honeycomb structure has large tensile strength, we note that it may buckle under compression. Given the atomic thin graphene wall in the carbon honeycomb and its low bending rigidity, the wall may be bent under compression, and give rise to a nonlinear stress-strain response during compression.

a

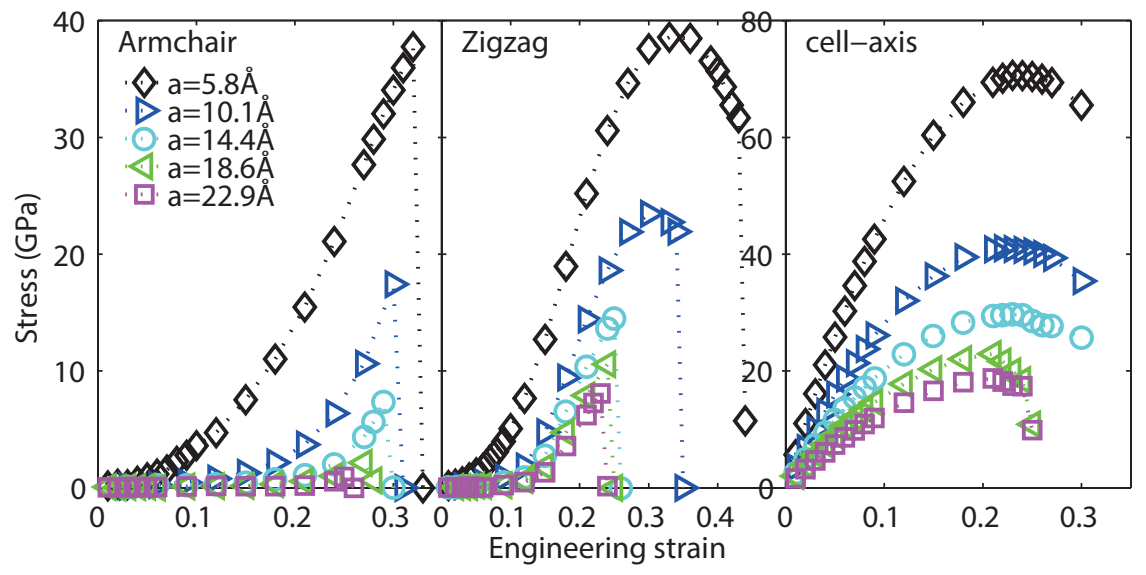

b

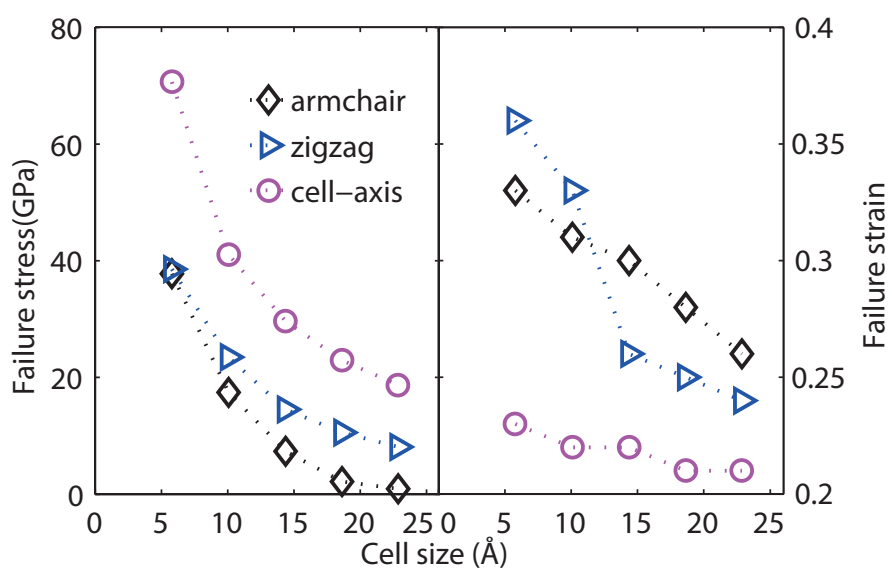

Figure 2. The mechanical behavior of C-honeycomb of different cell sizes. (a) Stress versus strain curves for tensile loading along the armchair (left), the zigzag (middle), and the cell-axis directions (right). (b) The failure strength (left) and failure strain (right) as a function of cell size. 
In addition to the appealing mechanical strengths in stable C-honeycomb, we also observe a strongly anisotropic Poisson's effect. Figure 3 shows the Poisson's ratio as a function of strain in C-honeycomb of different cell sizes. Following the convention, the Poisson's ratio $v_{a c-z z}$ defines the ratio of the resultant strain $\varepsilon_{z z}$ in the zigzag direction when a strain $\varepsilon_{a c}$ is imposed in the armchair direction, i.e., $v_{a c-z z}=-\varepsilon_{z z} / \varepsilon_{a c}$. In Figure $3 a$, we examine $v_{a c-z z}$ (left) and $v_{a c-z z}$ (right) as a function of strain. Tensile strain applied to the armchair (zigzag) direction of a C-honeycomb introduces nearly the same amount of compressive strain in the zigzag (armchair) direction. The Poisson's ratio can reach about 2 when the C-honeycomb reaches its failure strain. From the theoretical prediction for C-honeycombs, the Poisson's ratio for loading along the armchair direction is given $\operatorname{as}^{35} v_{a c-z z}=\frac{\cos ^{2} \theta}{(a / b+\sin \theta) \sin \theta}$; while that for loading along the zigzag direction is predicted to be $v_{z z-a c}=\frac{(a / b+\sin \theta) \sin \theta}{\cos ^{2} \theta}$. Given the hexagonal structure with $\theta=30^{\circ}$ at small deformation, $v_{a c-z z}=v_{z z-a c}=1$, which are in great agreement with DFT calculations shown in Figure 3. By looking at the subsequent deformation in the cell axis direction (z-direction in Figure 1a), we see that straining along the armchair or zigzag direction of a C-honeycomb introduces nearly no deformation along the cell-axis (Figure $3 b$ ), i.e., $v_{a c-z}$ and $v_{z z-z}$ are nearly zero. This anisotropic property could be utilized in functional structure design. For example, we may attach a layer of C-honeycomb material to either the inner or the outer wall of a tube with co-axial orientation of C-honeycomb with the tube. The C-honeycomb could then accommodate significant radius change in the tube without length change from the $\mathrm{C}$-honeycomb along 
the tube axis. Such structures may find their applications in biomedical engineering like expanding blood vessels radically without exerting any axial stretch.

a

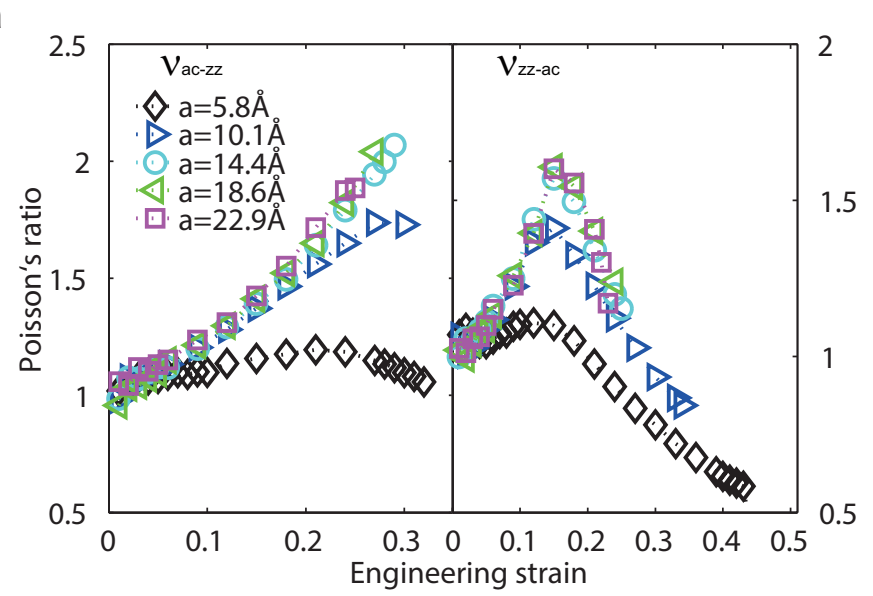

b

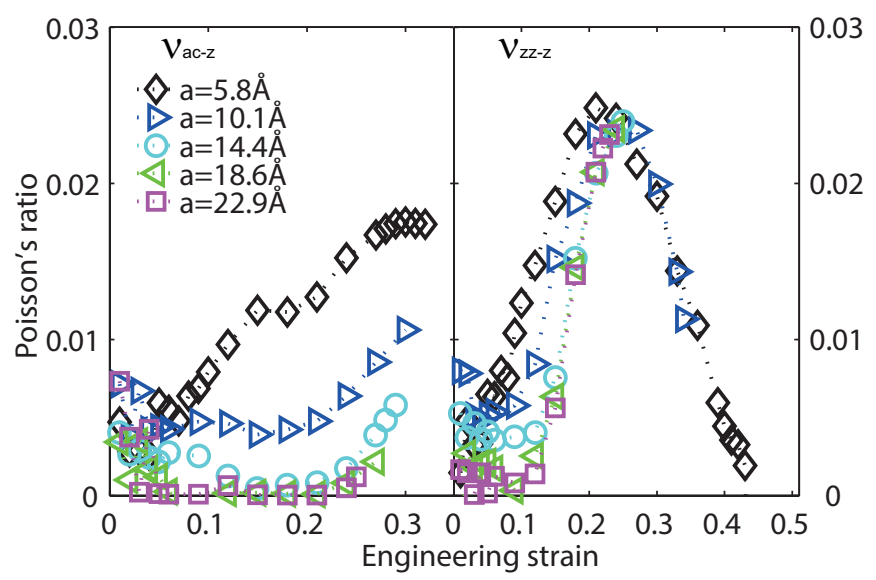

Figure 3. The strong anisotropic Poisson's effect in C-honeycomb of different cell sizes. (a) The Poisson's ratio versus strain curves for tensile loading along the armchair (left, $v_{\mathrm{ac}-\mathrm{zz}}$ ) and the zigzag direction (right, $v_{\mathrm{zz}-\mathrm{ac}}$ ). (b) The Poisson's ratio along the hexagonal tube direction (cell-axis) while loading along the armchair (left, $v_{\mathrm{ac}-\mathrm{z}}$ ) and zigzag-directions (right, $v_{\mathrm{zz}-\mathrm{z}}$ ).

One of the important feature of C-honeycomb is its ultra-light weight with tunable cell sizes that may be employed as electrodes for batteries with ultrafast charge and discharge rates ${ }^{37}$, flexible supercapacitor electrodes ${ }^{38}$, and storage media ${ }^{39}$. The density 
of C-honeycombs with $a=5.8 \AA$ to $29.7 \AA$ are only $1.31 \mathrm{~g} / \mathrm{cm}^{3}$ to $0.31 \mathrm{~g} / \mathrm{cm}^{3}$, much lower than $3.52 \mathrm{~g} / \mathrm{cm}^{3}$ for diamond and $2.26 \mathrm{~g} / \mathrm{cm}^{3}$ for graphite ${ }^{40}$. The high specific strength of 3-D C-honeycomb render it extremely competitive for the use as an ultra-light weight architected functional material ${ }^{41}$.

a

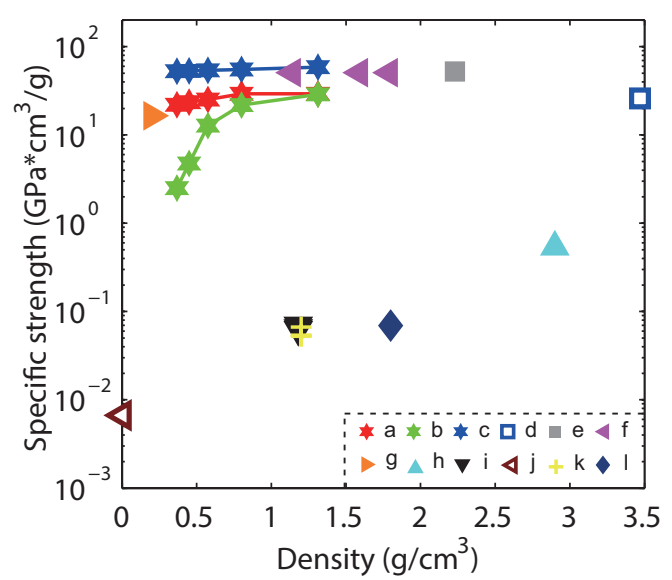

b

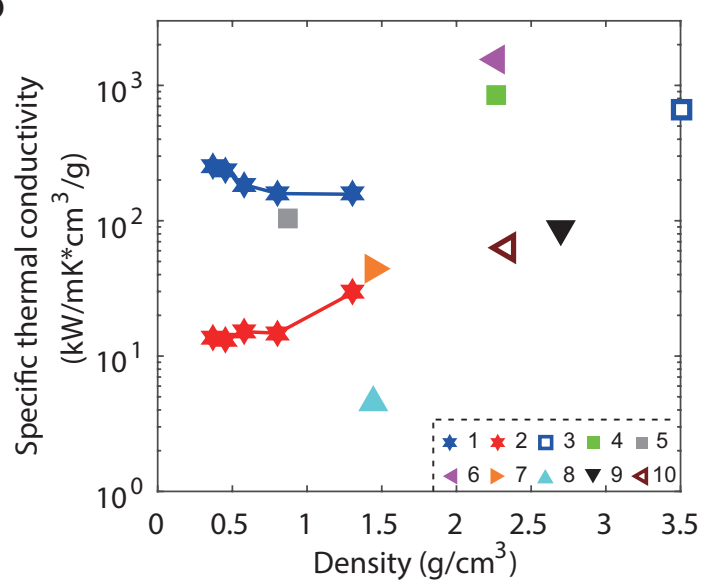

Figure 4. Specific strength and thermal conductivity of C-honeycomb. (a) Specific strength of $\mathrm{C}$-honeycomb of different cell sizes and other carbon-based materials. Here ' $a$ ' to 'm' refer to different data sources: ' $a$ ', 'b' and ' $c$ ' are, respectively, the specific strength of C-honeycomb along the zigzag, armchair and axial direction; ' $d$ ': diamond ${ }^{21}$; 'e': graphene ${ }^{3}$; 'f': CNTs of radius (3 3), (5 5), and (10 10) $)^{3}$; 'g': carbon-nanotube fiber'; ' $h$ ': ceramic nanolattices ${ }^{20}$; 'l': SWNT- MWNT- and FGS-PMMA ${ }^{22}$; 'j': ultra-flyweight aerogels $^{23}$; 'k': nanocomposite-SWNT -MWNT and -GPL ${ }^{24}$; 'l': graphene oxide paper ${ }^{10}$. (b) Thermal conductivity of carbon allotropes, their derivatives and some common materials. Here ' 1 ' to '10' refer to different data source: '1' and '2' are the specific thermal conductivity of c-honeycomb along cell axis and armchair (zigzag) direction, respectively; '3': diamond ${ }^{25}$; '4': graphite $^{25}$; '5': CNT arrays ${ }^{26}$; '6': graphene ${ }^{27}$; '7': graphene laminate ${ }^{28}$; '8': graphite nanoplatelet-epoxy composite ${ }^{29}$; '9': aluminum ${ }^{25}$; '10': silicon $^{25}$.

We show in Figure 4 the superior mechanical and thermal properties in comparison with other known low-density materials. As seen in Figure 4a, C-honeycomb trails to carbon nanotubes (CNTs) and graphene in terms of specific strength, rendering a 3-D material with much superior strengths than composites made of CNT or graphene ${ }^{3,}$ 
9, 10, 41-45. The specific thermal conductivity of C-honeycomb is presented in Figure $4 b^{46-50}$. SI-3, Figure S6 and Figure S7 describe the details about the calculation of the thermal conductivity of C-honeycombs with different sizes at room temperature using equilibrium molecular dynamics simulations as well as the obtained thermal conductivity data. The thermal conductivity along cell axis (armchair or zigzag) direction of C-honeycomb with $a=5.8 \AA$ is $205 \pm 60(39 \pm 11) \mathrm{W} / \mathrm{mK}$, and decreases to $96 \pm 25(5 \pm 2) \mathrm{W} / \mathrm{mK}$ when $a=22.9 \AA$. The thermal conductivity also exhibits strong anisotropy (Figure S7). Due to the ultra-low density and high thermal conductivity along the $z$ direction, the specific thermal conductivity of C-honeycombs is larger than most engineering materials, including metals and high thermal conductivity semiconductors, as well as light-weight CNT arrays ${ }^{47}$ and graphene-based nanocomposites ${ }^{50}$.

As mentioned at the beginning, the heterogeneous bonding in scaling up low-dimensional carbon allotropes as nanocomposites are intrinsically ineffective in reaching the properties that we desire to inherit from individual CNTs and graphene sheets, which limits the technological applications of those carbon allotropes. Here we demonstrate the feasibility of constructing stable 3-D architectured C-honeycomb with covalent bonding. The specific strength of C-honeycomb could be the best in structural carbon materials. Its specific thermal conductivity is also much better than most metal and high thermal conductivity semiconductors. Its strong anisotropic Poisson's effect may be utilized to design multi-functional structures with applications ranging from biomedical engineering to energy and environment systems. With the growing interest for 3-D nano-architectured functional materials, the well patterned two-level hexagonal 
structures in C-honeycomb pave a new strategy in achieving desirable properties that are comparable with carbon allotropes.

\section{Acknowledgements}

Y.W. acknowledges the support from National Natural Science Foundation of China (NSFC) (11425211). R.Y. acknowledges the support from the US National Science Foundation (Grant No. 1512776) along with the Teets Family Endowed Doctoral Fellowship to X.G. M.D. acknowledges the support from the US National Science Foundation (Grant No. 1507806). The calculations are performed at both the Supercomputing Center of CAS, and the Janus supercomputer, which is supported by the National Science Foundation (Grant No. 0821794).

Supporting Information. Simulation details of DFT calculations to determine the C-honeycomb structure, DFT calculations for the mechanical properties and MD simulations for the thermal conductivity.

\section{References}

1. Saito, R.; Dresselhaus, G.; Dresselhaus, M. S., Physical properties of carbon nanotubes. World Scientific: 1998; Vol. 35.

2. Novoselov, K. S.; Geim, A. K.; Morozov, S. V.; Jiang, D.; Zhang, Y.; Dubonos, S. V.; Grigorieva, I. V.; Firsov, A. A. Science 2004, 306, (5696), 666-669.

3. Lee, C.; Wei, X.; Kysar, J. W.; Hone, J. Science 2008, 321, (5887), 385-388.

4. Huang, P. Y.; Ruiz-Vargas, C. S.; van der Zande, A. M.; Whitney, W. S.; Levendorf, M. P.; Kevek, J. W.; Garg, S.; Alden, J. S.; Hustedt, C. J.; Zhu, Y. Nature 2011, 469, (7330), 389-392.

5. Lee, G.-H.; Cooper, R. C.; An, S. J.; Lee, S.; van der Zande, A.; Petrone, N.; Hammerberg, A. G.; Lee, C.; Crawford, B.; Oliver, W. Science 2013, 340, (6136), 1073-1076.

6. Grantab, R.; Shenoy, V. B.; Ruoff, R. S. Science 2010, 330, (6006), 946-948.

7. Wei, Y.; Wu, J.; Yin, H.; Shi, X.; Yang, R.; Dresselhaus, M. Nature Materials 2012, 11, (9), 759-763. 
8. Rasool, H. I.; Ophus, C.; Klug, W. S.; Zettl, A.; Gimzewski, J. K. Nature Communications 2013, 4.

9. Zhang, X.; Li, Q.; Holesinger, T. G.; Arendt, P. N.; Huang, J.; Kirven, P. D.; Clapp, T. G.; DePaula, R. F.; Liao, X.; Zhao, Y. Advanced Materials 2007, 19, (23), 4198-4201.

10. Dikin, D. A.; Stankovich, S.; Zimney, E. J.; Piner, R. D.; Dommett, G. H.; Evmenenko, G.; Nguyen, S. T.; Ruoff, R. S. Nature 2007, 448, (7152), 457-460.

11. Kim, P.; Shi, L.; Majumdar, A.; McEuen, P. Physical Review Letters 2001, 87, (21), 215502. 12. Marconnet, A. M.; Panzer, M. A.; Goodson, K. E. Reviews of Modern Physics 2013, 85, (3), 1295.

13. Karfunkel, H. R.; Dressler, T. Journal of the American Chemical Society 1992, 114, (7), 2285-2288.

14. Umemoto, K.; Saito, S.; Berber, S.; Tománek, D. Physical Review B 2001, 64, (19), 193409.

15. Kawai, T.; Okada, S.; Miyamoto, Y.; Oshiyama, A. Physical Review B 2005, 72, (3), 035428.

16. Kuc, A.; Seifert, G. Physical Review B 2006, 74, (21), 214104.

17. Zhu, Z.; Tománek, D. Physical Review Letters 2012, 109, (13), 135501.

18. Zhao, Z.; Xu, B.; Wang, L.-M.; Zhou, X.-F.; He, J.; Liu, Z.; Wang, H.-T.; Tian, Y. ACS Nano 2011, 5, (9), 7226-7234.

19. Enyashin, A. N.; Ivanovskii, A. L. Physica Status Solidi (b) 2011, 248, (8), 1879-1883.

20. Zhu, Z.; Fthenakis, Z. G.; Guan, J.; Tománek, D. Physical Review Letters 2014, 112, (2), 026803.

21. Park, N.; Ihm, J. Physical Review B 2000, 62, (11), 7614.

22. Fthenakis, Z. G. Molecular Physics 2013, 111, (21), 3289-3296.

23. Fahy, S.; Louie, S. G.; Cohen, M. L. Physical Review B 1986, 34, (2), 1191.

24. Fthenakis, Z. G. RSC Advances 2016, 6, (81), 78187-78193.

25. Krainyukova, N. V.; Zubarev, E. N. Physical Review Letters 2016, 116, (5), 055501.

26. Kresse, G.; Furthmüller, J. Computational Materials Science 1996, 6, (1), 15-50.

27. Kresse, G.; Furthmüller, J. Physical Review B 1996, 54, (16), 11169.

28. Blöchl, P. E. Physical Review B 1994, 50, (24), 17953.

29. Perdew, J. P.; Burke, K.; Ernzerhof, M. Physical Review Letters 1996, 77, (18), 3865-3868.

30. Togo, A.; Tanaka, I. Scripta Materialia 2015, 108, 1-5.

31. Guan, J.; Liu, D.; Zhu, Z.; Tománek, D. Nano Letters 2016, 16, (5), 3247-3252.

32. Plimpton, S. Journal of Computational Physics 1995, 117, (1), 1-19.

33. Kubo, R.; Toda, M.; Hashitsume, N., Statistical physics II: nonequilibrium statistical mechanics. Springer Science \& Business Media: 2012; Vol. 31.

34. Lindsay, L.; Broido, D. Physical Review B 2010, 81, (20), 205441.

35. Gibson, L. J.; Ashby, M. F., Cellular solids: structure and properties. Cambridge university press: 1999.

36. Wei, Y.; Wang, B.; Wu, J.; Yang, R.; Dunn, M. L. Nano Letters 2013, 13, (1), 26-30.

37. Li, N.; Chen, Z.; Ren, W.; Li, F.; Cheng, H.-M. Proceedings of the National Academy of Sciences 2012, 109, (43), 17360-17365.

38. Cheng, Y.; Lu, S.; Zhang, H.; Varanasi, C. V.; Liu, J. Nano Letters 2012, 12, (8), 4206-4211.

39. Patchkovskii, S.; John, S. T.; Yurchenko, S. N.; Zhechkov, L.; Heine, T.; Seifert, G. Proceedings of the National Academy of Sciences of the United States of America 2005, 102, (30), 10439-10444. 
40. Pierson, H. O., Handbook of carbon, graphite, diamonds and fullerenes: processing, properties and applications. William Andrew: 2012.

41. Meza, L. R.; Das, S.; Greer, J. R. Science 2014, 345, (6202), 1322-1326.

42. Telling, R.; Pickard, C.; Payne, M.; Field, J. Physical Review Letters 2000, 84, (22), 5160.

43. Ramanathan, T.; Abdala, A.; Stankovich, S.; Dikin, D.; Herrera-Alonso, M.; Piner, R.; Adamson, D.; Schniepp, H.; Chen, X.; Ruoff, R. Nature Nanotechnology 2008, 3, (6), 327-331.

44. Sun, H.; Xu, Z.; Gao, C. Advanced Materials 2013, 25, (18), 2554-2560.

45. Rafiee, M. A.; Rafiee, J.; Wang, Z.; Song, H.; Yu, Z.-Z.; Koratkar, N. ACS Nano 2009, 3, (12), 3884-3890.

46. Ho, C. Y.; Powell, R. W.; Liley, P. Journal of Physical and Chemical Reference Data 1972, 1, (2), 279-421.

47. Cola, B. A.; Xu, X.; Fisher, T. S. Applied Physics Letters 2007, 90, (9), 093513.

48. Chen, S.; Wu, Q.; Mishra, C.; Kang, J.; Zhang, H.; Cho, K.; Cai, W.; Balandin, A. A.; Ruoff, R. S. Nature materials 2012, 11, (3), 203-207.

49. Malekpour, H.; Chang, K.-H.; Chen, J.-C.; Lu, C.-Y.; Nika, D.; Novoselov, K.; Balandin, A. Nano Letters 2014, 14, (9), 5155-5161.

50. Yu, A.; Ramesh, P.; Itkis, M. E.; Bekyarova, E.; Haddon, R. C. The Journal of Physical Chemistry C 2007, 111, (21), 7565-7569. 


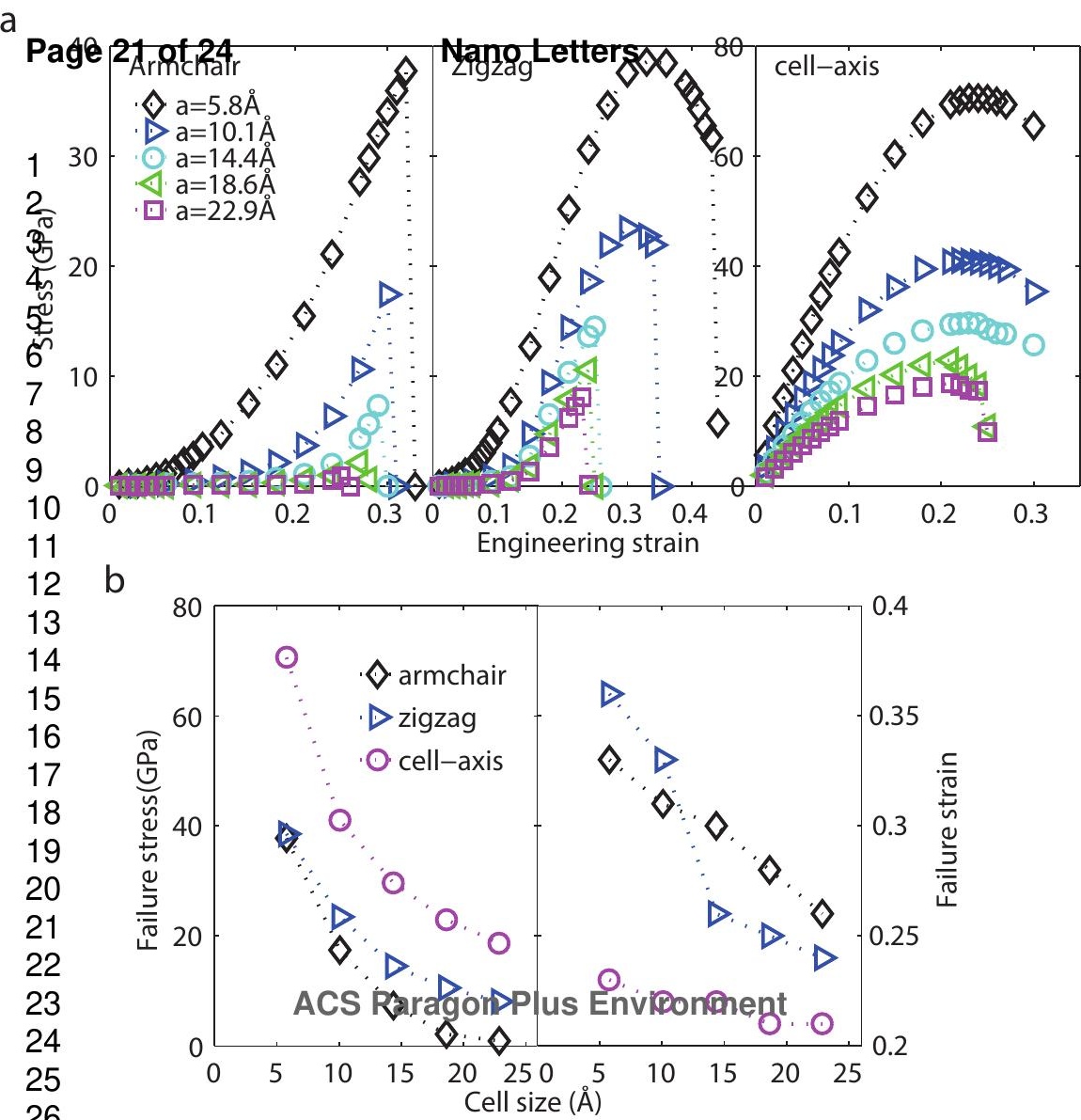

a 


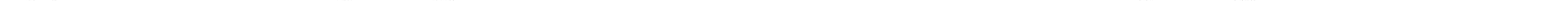




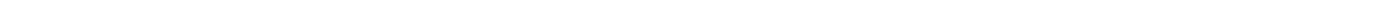




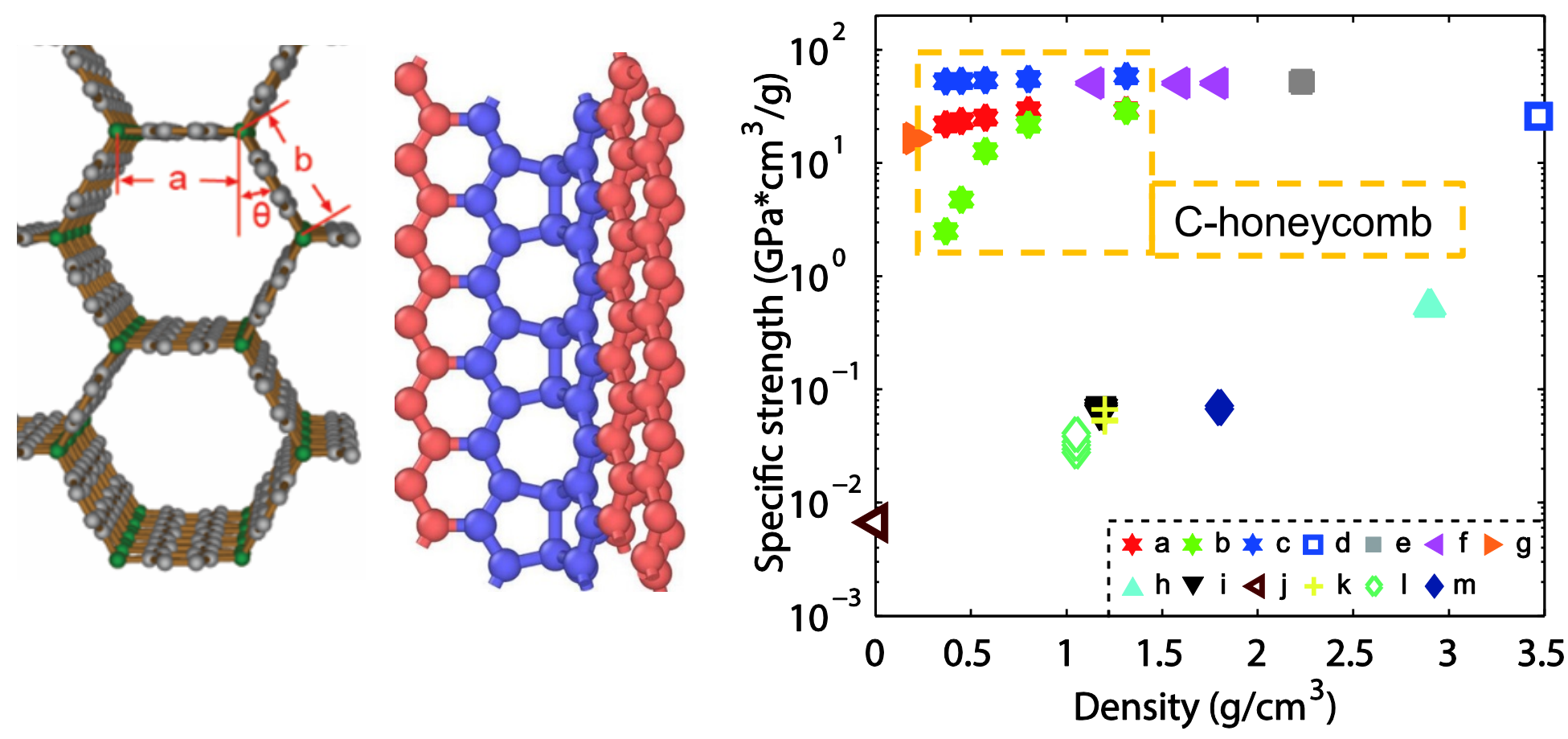

